\title{
Benefits and challenges of standard ceiling-mounted surface guided radiotherapy systems for breast treatments on Halcyon ${ }^{\mathrm{TM}}$
}

\author{
D. Nguyen ${ }^{1, *},{ }^{\infty}$, J. Farah $^{2}$, F. Josserand-Pietri ${ }^{1}$, N. Barbet ${ }^{1}$ and M. Khodri ${ }^{1}$ \\ ${ }^{1}$ ORLAM Group, Department of Radiation Oncology, 44 rue Ambroise Paré, 71000 Mâcon, France. \\ 2 Vision RT Ltd., Dove House, Arcadia Avenue, London N3 2JU, United Kingdom.
}

Received: 26 March 2021 / Accepted: 6 September 2021

\begin{abstract}
The present work investigates the benefits and challenges of applying surface guided radiation therapy (SGRT) to breast cancer treatments on the Halcyon ${ }^{\mathrm{TM}}$ (Varian, USA). Inter-fraction setup accuracy and treatment time were assessed by comparing CBCT shifts registered following SGRT-based $v s$. standard tattoo-based patient setup in two randomly selected groups of ten breast patients. Next, using a torso phantom and a volunteer, intra-fraction motion tracking accuracy and surface coverage were assessed for 3 adjusted central ceiling camera positions. SGRT was shown to significantly reduce setup errors (mostly within $\pm 2 \mathrm{~mm}$ ) compared to tattoos (up to $2.1 \mathrm{~cm}$ ) in all translational directions ( $p$-value $<0.001$ ). Treatment throughput and efficiency were also significantly improved with SGRT $(p=0.038)$. Meanwhile, intra-bore surface coverage with an adjusted central SGRT camera proved insufficient, suffering from patient self-occlusions (invisible body parts occluded by patients' own morphology such as breasts, bellies, arms, etc.) and bore-induced camera obstructions. Tracking accuracy remained satisfactory (sub- $0.5 \mathrm{~mm}$ ) but 6 degrees-of-freedom motion monitoring, critical in stereotactic radiosurgery, stereotactic body radiation therapy and deep inspiration breath hold techniques and clinical applications, was not possible. Standard ceiling-mounted SGRT systems reduce inter-fraction breast setup errors and treatment duration while intra-fraction motion tracking is insufficient for O-ring linear accelerators.
\end{abstract}

Keywords: breast cancer / Halcyon ${ }^{\mathrm{TM}}$ / surface guidance / patient positioning / treatment time / intra-bore tracking

\section{Introduction}

Recent years have witnessed the development of surface guided radiation therapy (SGRT) as a means for fast and accurate patient setup, live and continuous monitoring of intrafraction patient motion and increased treatment efficiency and throughput (Li et al., 2011; Herron et al., 2018; Kost et al., 2019).

SGRT has been used with various delivery techniques (for example protons, photons: conformal/conventional, intensity modulated radiotherapy, and volumetric modulated arc therapy) and for all anatomical sites including standard intracranial, torso and pelvic applications. SGRT has also been associated with various advanced techniques such as deep inspiration breath hold(DIBH), stereotactic radiosurgery (SRS), stereotactic body irradiations (SBRT), etc. showing

\footnotetext{
*Corresponding author: Nguyen.daniel@yahoo.fr

a Present address: Daniel Nguyen, Lead Medical Physicist, Groupe ORLAM Mâcon, Polyclinique du Val de Saône, 44 rue Ambroise Paré, Mâcon, France.
}

clinical outcomes (local/regional tumor control, actuarial survival rates, radiation-induced toxicities) equivalent to image-guided radiotherapy (Li et al., 2013; Pham et al., 2014; Batin et al., 2016; Zagar et al., 2017; Heinzerling et al., 2020).

However, the use of such technology on closed-bore linear accelerators such as Halcyon ${ }^{\mathrm{TM}}$ and Ethos ${ }^{\mathrm{TM}}$ (Varian Medical Systems Inc., Palo Alto, USA) or TomoTherapy (Accuray, Sunnyvale, USA) remains scarce. In fact, a recent publication reported the use of SGRT for initial patient setup on Halcyon for non-SRS intracranial treatments showing faster setup and treatment time along with a $50 \%$ reduction of repeat imaging (Flores-Martinez et al., 2020). However, benefits of SGRT for other clinical sites such as breast, one of the most widely treated cancer on Halcyon ${ }^{\mathrm{TM}}$ accelerators, have not been previously reported. Meanwhile, for TomoTherapy units, SGRT based positioning performed better than laser-based positioning while inter-operator variability and the uncertainty associated with the movement of the patient during MVCT image acquisition as well as during image registration and analysis ultimately yielded equivalent total MVCT accuracy for both methods (Crop et al., 2016). Authors also concluded 
Table 1. Original and adjusted central camera positions with respect to treatment isocenter.

\begin{tabular}{|c|c|c|c|c|}
\hline \multicolumn{2}{|c|}{ Original central camera position (tilted towards setup isocenter, i.e. bore lasers) } & \multicolumn{3}{|c|}{ Adjusted central camera positions } \\
\hline Vertical $(\mathrm{cm})$ & 139.33 & 139 & 106.3 & 116.1 \\
\hline Longitudinal $(\mathrm{cm})$ & 217.64 & 217.64 & 166.1 & 166.1 \\
\hline Lateral $(\mathrm{cm})$ & 0.06 & 0.06 & 0.06 & 0.06 \\
\hline Tilt $\left({ }^{\circ}\right)$ & 29.5 & 50.3 & 50.3 & 45.7 \\
\hline
\end{tabular}

that real-time monitoring is required to reduce important intrafraction movement.

Additionally, to the best of authors' knowledge, the literature still lacks any publication investigating the performance of SGRT in tracking intra-bore/intra-fraction patient motion on O-ring linear accelerators. This is particularly relevant and critical for online adaptive planning and delivery on Ethos ${ }^{\mathrm{TM}}$ and the latest Radixact ${ }^{\circledR}$ TomoTherapy units, as well as to monitor breath-hold stability for DIBH (and Deep Exhale Breath Hold) treatments. In fact, a recent paper reported the use of an in-house camera mounted on the back wall of the treatment vault to track 3D-printed anthropomorphic phantoms representing a head, hand and breast; positioned at the Halcyon ${ }^{\text {TM }}$ isocenter (Delombaerde et al., 2019). Although the study proved intra-bore surface scanning feasible in an O-ring bore, several limitations can be highlighted preventing routine clinical use. Namely, system accuracy could not reach the sub-millimetric level of standard SGRT solutions, surface coverage and quality showed deviations and limited lateral coverage (for the head phantom), patient self-occlusion was not investigated (breast torso phantom excluded head/ arm) and the latency time $(\sim 1 \mathrm{~s})$ proved incompatible with breathing motion detection.

This work hence investigates the benefits of SGRT for patient setup and inter-fraction motion management in breast cancer radiotherapy on Halcyon ${ }^{\mathrm{TM}}$. Additionally, experiments with different central camera positions were performed to assess surface coverage, accuracy and possible challenges for standard ceiling mounted SGRT solutions for intra-fraction motion monitoring considering a female torso phantom and a volunteer.

\section{Methods}

\subsection{SGRT for patient setup on Halcyon ${ }^{\mathrm{TM}}$}

The Halcyon ${ }^{\mathrm{TM}}$ treatment platform contains a $6 \mathrm{MV}$ flattening filter free (FFF) linear accelerator mounted on a $1 \mathrm{~m}$ wide O-ring gantry allowing for fast and accurate intensity modulated and volumetric arc radiotherapy (IMRT and VMAT) treatments thanks to a nominal dose rate of $800 \mathrm{MU} / \mathrm{min}$, a $17 \mathrm{~s}$ long cone-beam CT (CBCT) imaging, and a dual-layer multi-leaf collimator (MLC). The clinical workflow with Halcyon ${ }^{\mathrm{TM}}$ includes setting up the patient outside the bore at the virtual isocenter (i.e. bore-mounted lasers), automatic couch shift to the treatment isocenter and mandatory daily imaging before treatment delivery. The sitespecific automatic couch transition from virtual to treatment isocenters are defined during system calibration, and at Groupe ORLAM Mâcon involve shifts of $0.33 \mathrm{~cm}, 57.64 \mathrm{~cm}$ and $0.06 \mathrm{~cm}$ in vertical, longitudinal and lateral directions respectively.

To enable SGRT-guided patient setup on Halcyon ${ }^{\mathrm{TM}}$, three AlignRT (Vision RT Ltd., London, UK) camera units (pods) are typically installed at $\pm 155 \mathrm{~cm}$ lateral, $-80 \mathrm{~cm}$ longitudinal and $135 \mathrm{~cm}$ vertical for left/right camera pods and $160 \mathrm{~cm}$ longitudinal and $139 \mathrm{~cm}$ vertical for the central camera. These distances are defined in relation to the virtual isocenter and cameras were calibrated to this position.

The accuracy and benefits of SGRT in patient setup were quantified by retrospectively analysing CBCT shifts obtained for ten randomly selected breast patients tracked for ten sessions each, using SGRT for initial patient setup at the virtual isocenter. These CBCT shifts were compared against values observed when ten other patients were positioned using lasers and tattoos for ten sessions each. Student t-test was performed to investigate statistically significant improvement of patient setup and treatment time. Postural alignment of the patient including overall body/torso rotations, arm and chin setup were also tracked and compared between SGRT setup and tattoos and laser setup. Finally, patient setup time was also noted to determine any time sparing as a secondary benefit of SGRT.

\subsection{Intra-bore tracking}

Additionally, to track intra-fraction patient motion, the position and orientation of the central ceiling camera was adjusted to the treatment isocenter as documented in Table 1. The quality and coverage/extent of the surface obtained by the central camera pod was determined using a female torso phantom (The Competitive Store, Santa Ana, USA). This moulded plastic phantom includes fully featured front half form with open back and following dimensions: height $58.4 \mathrm{~cm}$, shoulder width $39.4 \mathrm{~cm}$, chest width $25.8 \mathrm{~cm}$, hip width $30.5 \mathrm{~cm}$, depth $14 \mathrm{~cm}$ and a breast size of $34 \mathrm{C}$. A BreastBoard LX ${ }^{\mathrm{TM}}$ (Macromedics, Moordrecht, The Netherlands) was used to simulate left breast cancer treatments at $0^{\circ}, 5^{\circ}, 7.5^{\circ}$ and $10^{\circ}$ inclinations. The accuracy of the one camera pod system was also tested by applying known couch shifts of $\pm 2 \mathrm{~cm}$ in vertical, longitudinal and lateral successively and in combination; additionally the breast board was used at three different heights to simulate pitch change of $2.5^{\circ}$ and $5^{\circ}$. Additional experiments were carried out with a volunteer to assess surface coverage considering different intracranial, chest and breast anatomical sites ( $c f$. Fig. 1). 

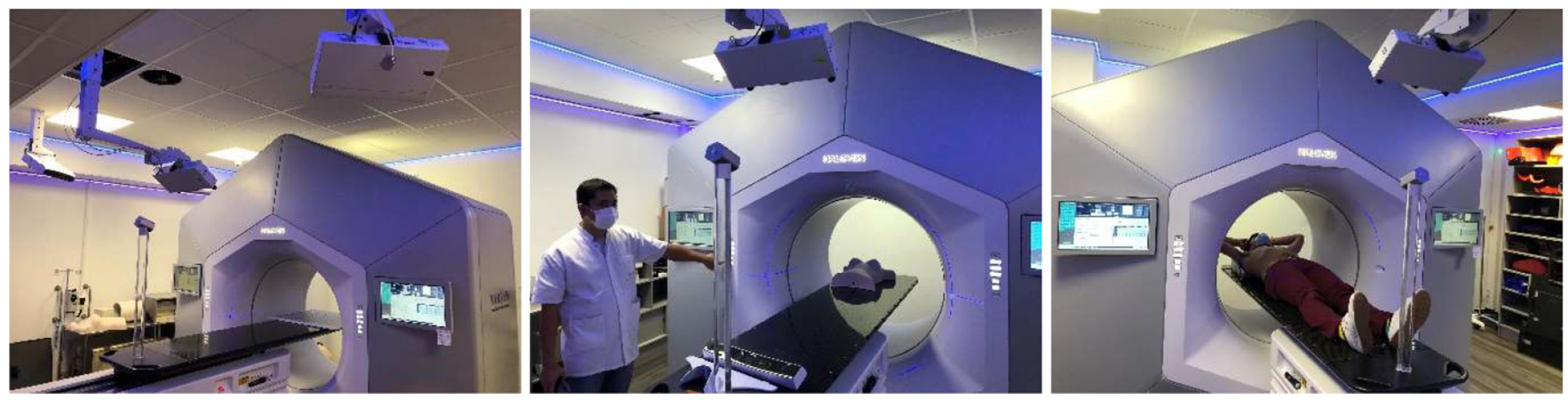

Fig. 1. Adjustment of central ceiling camera and calibration for intra-bore tracking (left), surface coverage and accuracy tests with a female torso phantom (middle) and a volunteer (right).

\section{Results and discussions}

\subsection{SGRT benefits for patient setup}

Figure 2 shows wide dispersion of $\mathrm{CBCT}$ shifts, also exceeding $2 \mathrm{~cm}$, when patient positioning is performed with the help of tattoos with most of the sessions (62\%) involving shifts exceeding $\pm 2 \mathrm{~mm}$. Meanwhile, following SGRT-guided patient setup, CBCT translational shifts were minimal with most of the sessions $(52 \%)$ requiring adjustments below $\pm 2 \mathrm{~mm}$. Comparison of CBCT shifts following tattoos or SGRT positioning are given in Table 2. Results of the statistical analysis are also given in Table 2 showing significant improvement of patient setup in all translational directions $(p<0.001)$. SGRT was also found to positively improve postural alignment of the patient (torso, arms, chin) eliminating gross rotational errors which may be frequent when solely using tattoos for patient setup and which cannot be corrected with the 3 DOF Halcyon ${ }^{\mathrm{TM}}$ treatment couch. Tracking the patient treatment time showed that SGRT offers significant $(p=0.038)$ time sparing compared to tattoos with treatment times of $10.9 \pm 1.4 v s .11 .3 \pm 2.1 \mathrm{~min}$ respectively ( $c f$. Tab. 2 ). Additionally, since breast patients represent $\sim 35 \%$ of the treatments performed on Halcyon ${ }^{\mathrm{TM}}$ at the local clinic, this increased throughput and treatment efficiency is highly appreciated. Finally, the largest time saving offered by SGRT can be attributed to the possibility of correcting rotational shifts prior to CBCT imaging. This allows for avoiding multiple patient setup attempts and repeat imaging ( $\mathrm{kV}$ or MV CBCT, MV ports, etc.), a situation which generally cannot be prevented with tattoos and the respective lack of $6 \mathrm{DoF}$ patient setup information relative to SGRT. Based on this work, it is also safe to conclude that tattoos avoidance is feasible for patient treatments on Halcyon ${ }^{\mathrm{TM}}$ improving thereby the patient quality of life post-radiotherapy while making no compromises on setup accuracy (Herron et al., 2018; Moser et al., 2020).

\subsection{SGRT challenges for intra-fraction motion management}

Experimental measurements with the female torso phantom show a limited surface coverage for all three adjusted ceiling mounted central camera positions, even with a breast board set to the largest $10^{\circ}$ inclination where the effects of
With SGRT

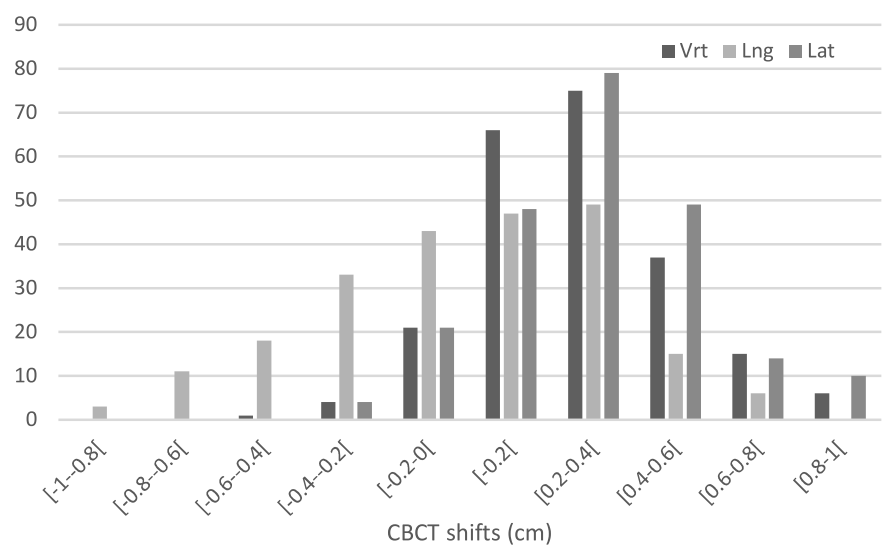

With Tattoos

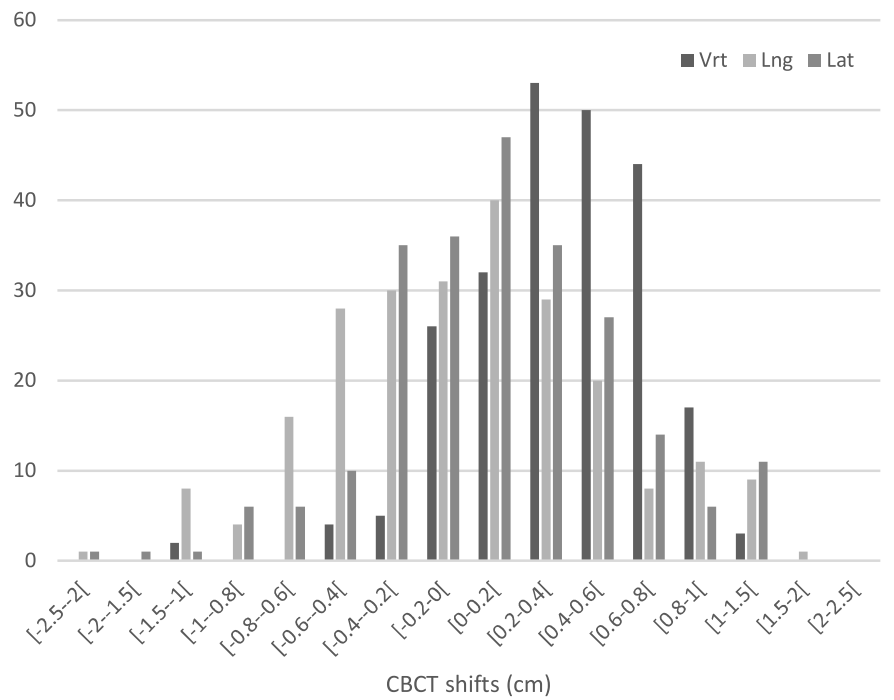

Fig. 2. Distribution of CBCT shifts after patient setup with tattoos (top) or SGRT (bottom).

patient self-occlusion were minimized ( $c f$. Fig. 3). The observed large holes in the surfaces are the result of selfocclusions caused by the phantom breasts and body morphology. Additionally, a one camera pod aligned to the treatment isocenter on the longitudinal axis proved to result in 
Table 2. Comparison of CBCT shifts and session time recorded following patient positioning with tattoos or SGRT.

\begin{tabular}{|l|l|l|l|l|l|}
\hline & & Vertical $(\mathrm{cm})$ & Longitudinal $(\mathrm{cm})$ & Lateral $(\mathrm{cm})$ & Session time $(\mathrm{min})$ \\
\hline \multirow{3}{*}{$\begin{array}{l}\text { CBCT shifts post tattoo } \\
\text { and laser setup (N=236) }\end{array}$} & $\begin{array}{l}\text { Average } \pm \text { standard } \\
\text { deviation }\end{array}$ & $0.3 \pm 0.4$ & $-0.1 \pm 0.6$ & $0.0 \pm 0.5$ & $11.3 \pm 2.1$ \\
\cline { 2 - 6 } & Median (min-max) & $0.3(-1.2-1.3)$ & $-0.1(-2.1-1.5)$ & $0.0(-2.1-1.4)$ & $11.3(6.9-14.4)$ \\
\cline { 2 - 6 } & Q1-Q3 & $0.10-0.60$ & $-0.4-0.3$ & $-0.3-0.3$ & $10.1-13.1$ \\
\hline \multirow{3}{*}{$\begin{array}{l}\text { CBCT shifts post SGRT } \\
\text { setup (N=225) }\end{array}$} & $\begin{array}{l}\text { Average } \pm \text { standard } \\
\text { deviation }\end{array}$ & $0.2 \pm 0.2$ & $-0.1 \pm 0.3$ & $0.3 \pm 0.2$ & $10.9 \pm 1.4$ \\
\cline { 2 - 6 } & Median (min-max) & $0.2(-0.5-0.9)$ & $0.0(-1.0-0.7)$ & $0.3(-0.4-0.9)$ & $11.2(7.0-12.7)$ \\
\cline { 2 - 6 } & Q1-Q3 & $0-0.4$ & $-0.3-0.2$ & $0.1-0.4$ & $10.0-12.0$ \\
\hline \multirow{2}{*}{$t$-test } & $p$-value & $<0.0001$ & $<0.0001$ & $<0.0006$ & 0.038 \\
\hline
\end{tabular}
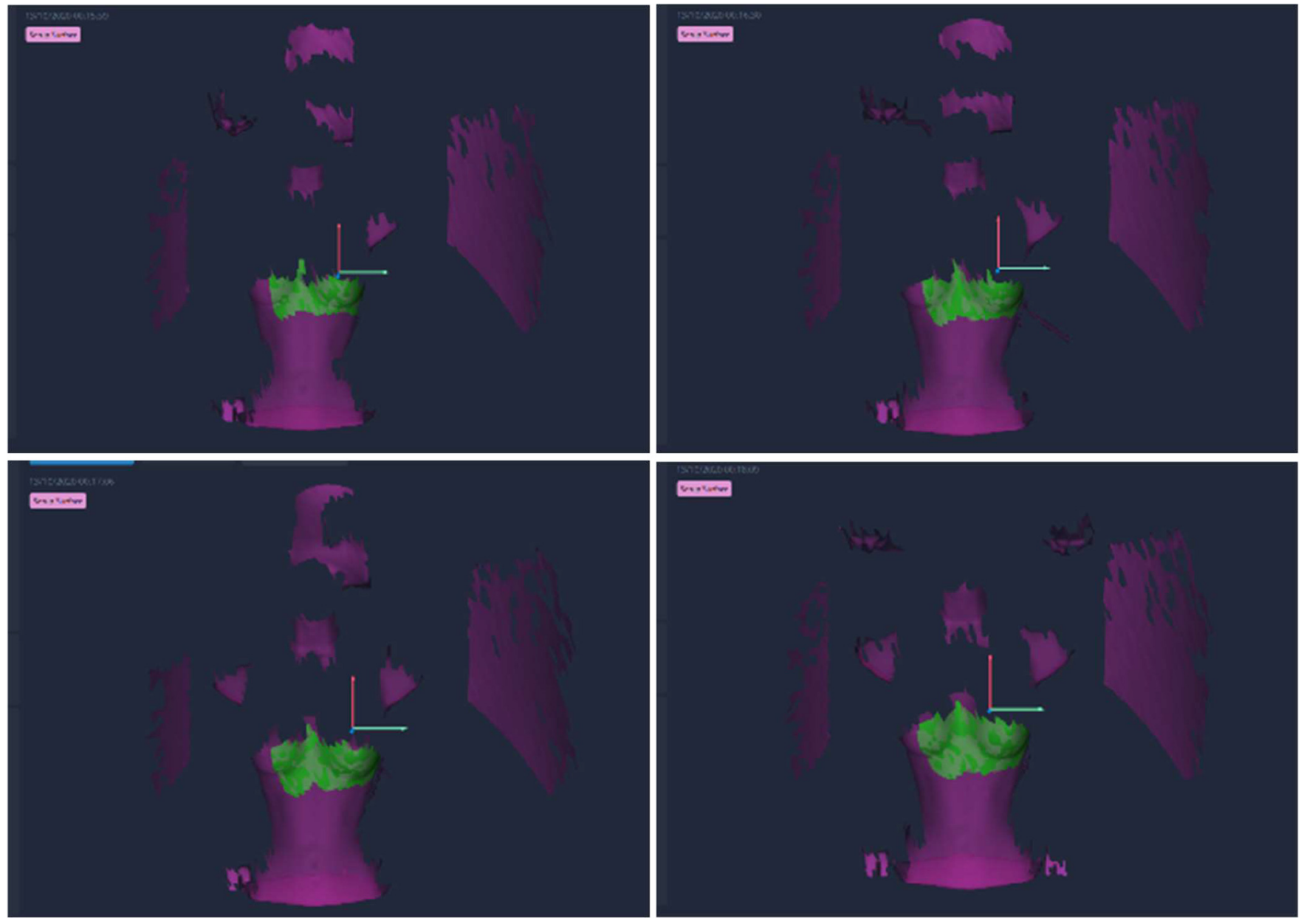

Fig. 3. Surface coverage obtained with the titled central ceiling camera monitoring the female torso phantom lying flat on the breast board (top left), on a $5^{\circ}$ inclination (top right), $7.5^{\circ}$ inclination (bottom left) and $10^{\circ}$ inclination (bottom right). 

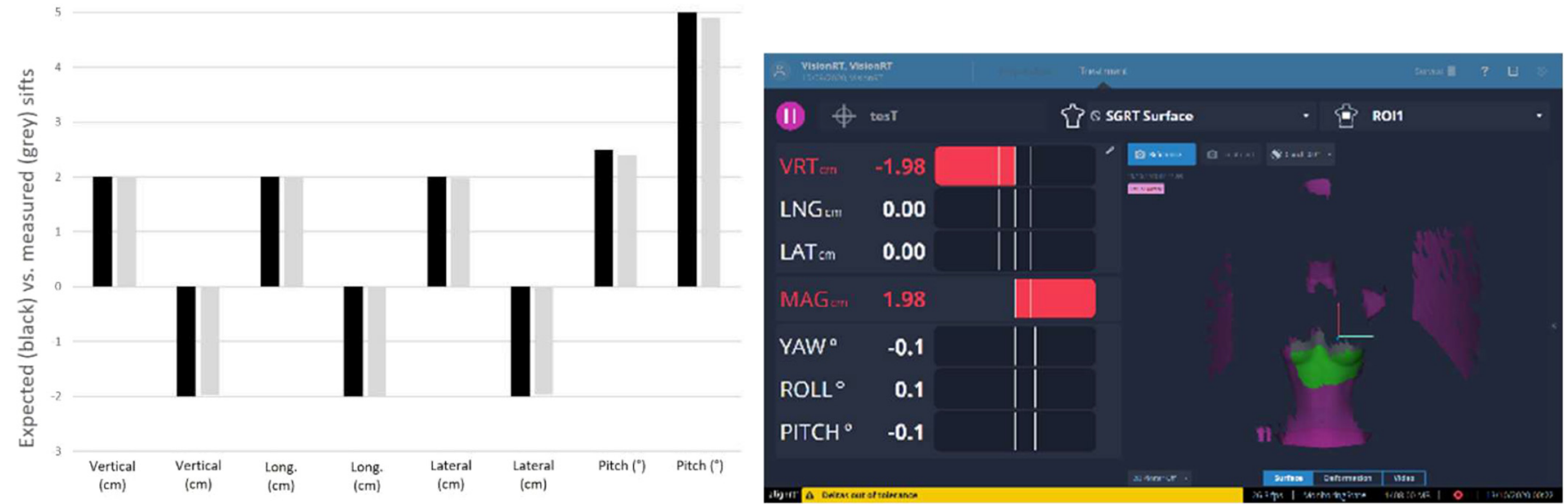

Fig. 4. Accuracy tests with the tilted central ceiling camera (Pos. \#1) monitoring the female torso phantom (left) and example of ROI used for surface tracking (right).
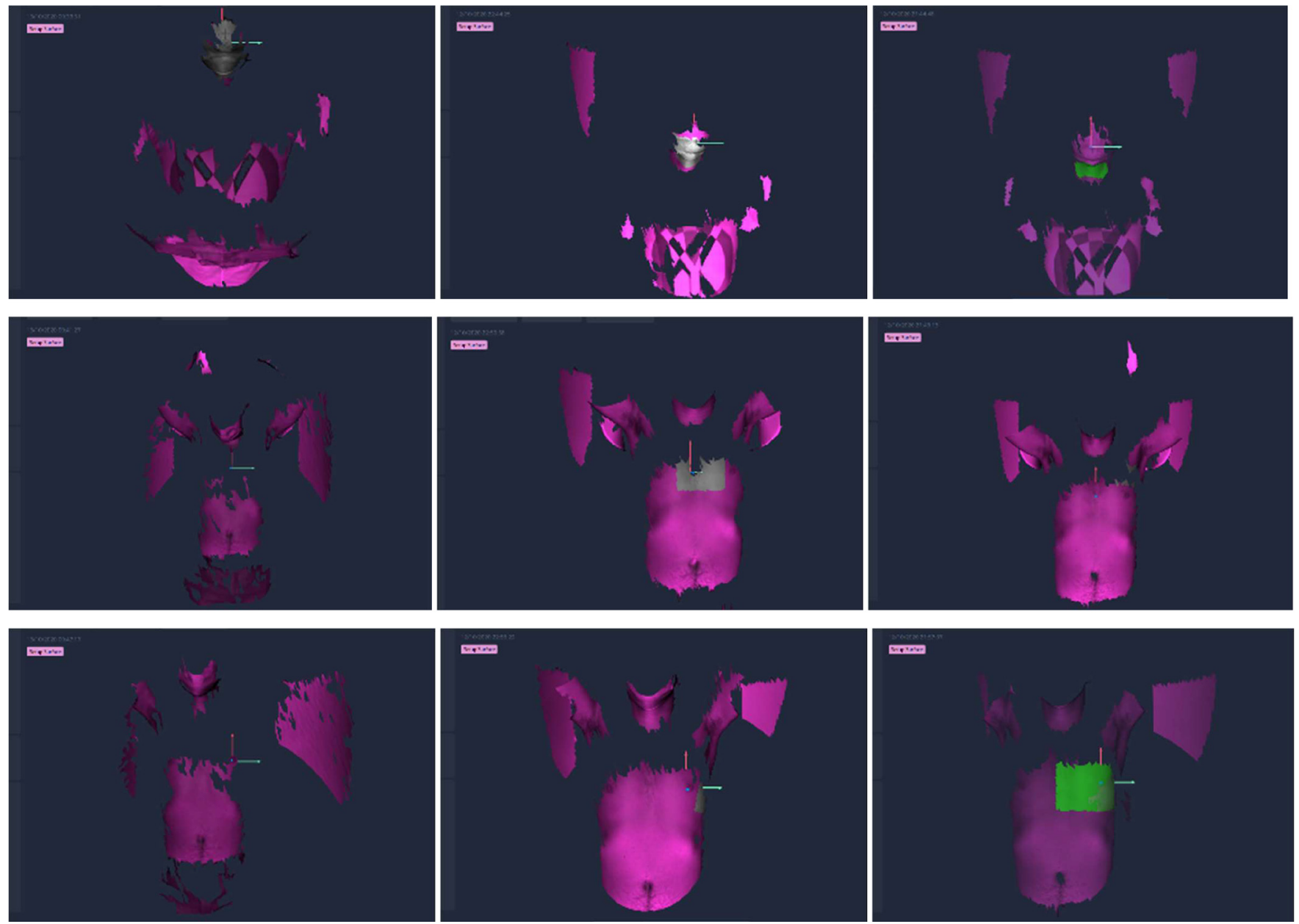

Fig. 5. Surface coverage obtained with the three adjusted central camera positions, tests performed on volunteer simulating intracranial (left), thorax (middle) and $5^{\circ}$ breast board setup (right). Adjusted central camera at position \#1 (top), \#2 (middle) and \#3 (bottom). 

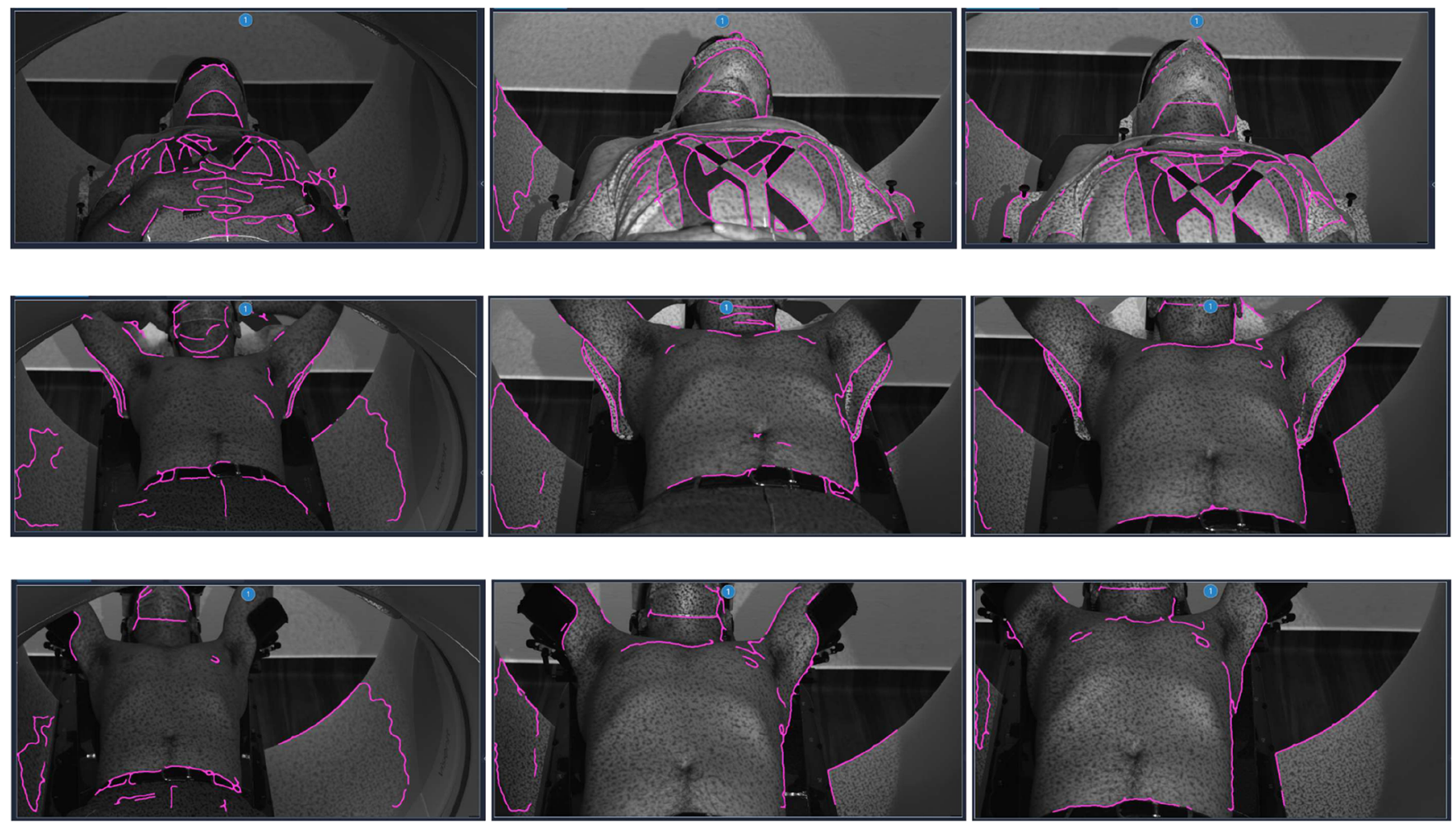

Fig. 6. Camera Line of site with the volunteer showing self-occlusions in intracranial (top), thorax (middle) and $5^{\circ}$ breast board setup (bottom). Adjusted central camera at position \#1 (left), \#2 (middle) and \#3 (right).

limited lateral surface coverage. Meanwhile, additional lateral pods would be susceptible to occlusions from the bore structure. Such an insufficient coverage and sub-optimal surface qualities would not allow for 6 degrees of freedom (6 DOF) tracking of intra-fraction patient motion as required for advanced treatments such as DIBH. Higher camera setup resulting in steep angulation and cleared line of site for the upper torso section of the phantom can help reduce patient self-occlusion but is limited by the bore structure obstructions. In opposition, lower camera positions would avoid any occlusions due to the bore structure but would suffer from limited (longitudinal) surface coverage due to patient selfocclusions of the cameras. Finally, camera positioning is also constrained by the elements of the ceiling (skylights, CCTV cameras, lasers, gallows, etc.), and risks of camera knocking if these were set too low.

Meanwhile, for all three adjusted central camera positions, tests with the female torso phantom proved SGRT to measure, with sub- $0.3 \mathrm{~mm}$ and sub $0.1^{\circ}$ accuracy, the mechanical shifts applied to the treatment couch and the used breast board inclination (cf. Fig. 4). This result however solely relies on the tracking of central surface elements while the typically recommended regions of interest with anatomical landmarks (breast, upper torso with supra-clavicles, lateral) are not available due to the occlusion limitations (Guo et al., 2017).

Lastly, tests with volunteer also confirm the previously observed results showing poor surface coverage for intracranial/head and neck applications (cf. Fig. 5). Insufficient information for chest and breast applications is also observed with the presence of holes (i.e. missing surface information) at the supra-clavicles and arms/chins (cf. Fig. 5). For pelvic locations, surface coverage seems reasonably sufficient but may be subject to greater occlusions for patients with a large abdomen/high body mass index (BMI) (cf. Fig. 4). Figure 6 represents the camera line of site with the volunteer clearly showcasing self-occlusions likely to be further increased with a female patient (breasts) or heavy/high BMI patients (belly). Based on Figures 3-6, it is safe to conclude that standard SGRT solutions would not allow for 6 DOF intrafraction motion tracking due to a limited line of sight, restrained lateral coverage and poor surface reconstruction of the upper torso, neck and head regions. Standard ceiling mounted cameras are also likely to suffer underperformance for heavy breathing patients, overweight patients with large bellies or breasts. Additionally, the observed poor surface coverage and patient self-occlusions are expected to be even more pronounced when the patient is instructed to take a deep inspiration - a technique widely used for left breast cancer treatment (Bergom et al., 2018). Specifically, when the inspiration is taken at the chest level (ideal situation), patient's breasts are likely to hide a larger portion of the upper torso and more specifically the supraclavicular section. Similarly, if the patient achieves breath hold while filling the abdomen, the mid-section of the torso is likely to be occluded to the cameras.

The present work also included some limitations. First, the number of selected patients to compare CBCT shifts following patient setup with tattoos or with SGRT is limited to two groups of ten patients each. However, with over 450 data points to compare, a robust statistical analysis could be performed demonstrating improved patient setup accuracy and 
throughput with surface guidance as observed in the abundant literature (Chang et al., 2012; Kost et al., 2019; Kügele et al., 2019). Additionally, experiments with an adjusted central camera position were limited to three different setup positions and could not simulate all possible scenarios. Nonetheless, challenges arising from bore occlusions and patient selfocclusions would not be overcome with additional ceiling mounted SGRT elements; this conclusion also holds for cameras on the rear side of the Halcyon ${ }^{\mathrm{TM}}$ bore (Delombaerde et al., 2019). Finally, surface coverage was tested with a female torso phantom and a single male volunteer. Although the study would beneficiate from a higher number of volunteers including females with different body morphology and breast size, it is safe to conclude that an adjusted central ceiling cameras position would not be sufficient to allow for accurate intra-fraction motion tracking with standard and clinically-relevant ROI for all clinical applications given the limited camera line of sight and the poor surface coverage observed with the male volunteer (Guo et al., 2017).

\section{Conclusion}

Compared to using skin tattoos, SGRT on Halcyon ${ }^{\mathrm{TM}}$ allows more accurate and faster breast patient setup while reducing the need for patient re-alignment and repeat imaging ( $\mathrm{kV}$ or MV CBCT, MV ports). Meanwhile, even with an adjusted central camera positioning, experimental measurements showed limited lateral surface coverage and poor surface qualities for 6 DOF intra-bore patient motion tracking with standard SGRT. Major challenges for ceiling mounted SGRT systems involve patient self-occlusions and bore obstructions. Instead, a dedicated SGRT solution for O-ring linear accelerators seems necessary.

\section{Conflict of interest}

Dr. Jad Farah is employed by Vision RT Ltd.

ORLAM group has signed a collaboration agreement with Vision RT Ltd. to develop the AlignRT InBore ${ }^{\text {TM }}$ platform.

\section{Acknowledgements}

Authors wish to thank the radiation therapists working at ORLAM/Mâcon for their valuable contribution to this work through patient data collection as well as Roch Deriaz and Philippe Mathieu, from Vision RT Ltd., for their support during the experimental measurements.

\section{References}

Batin E, Depauw N, MacDonald S, Lu H-M. 2016. Can surface imaging improve the patient setup for proton postmastectomy chest wall irradiation? Pract. Radiat. Oncol. 6(6): e235-e241.
Bergom C et al. 2018. Deep Inspiration Breath Hold: Techniques and advantages for cardiac sparing during breast cancer irradiation. Front Oncol. 8: 87. https://doi.org/10.3389/fonc.2018.00087.

Chang AJ et al. 2012. Video surface image guidance for external beam partial breast irradiation. Pract. Radiat. Oncol. 2(2): 97-105.

Crop F et al. 2016. Surface imaging, laser positioning or volumetric imaging for breast cancer with nodal involvement treated by helical Tomotherapy. J. Appl. Clin. Med. Phys. 17(5): 200-211.

Delombaerde L, Petillion S, Michiels S, Weltens C, Depuydt T. 2019. Development and accuracy evaluation of a single-camera intrabore surface scanning system for radiotherapy in an O-ring linac. Phys. Imag. Radiat. Oncol. 11: 21-26.

Flores-Martinez E, Cerviño LI, Pawlicki T, Kim G-Y. 2020. Assessment of the use of different imaging and delivery techniques for cranial treatments on the Halcyon linac. J. Appl. Clin. Med. Phys. 21(1): 53-61.

Guo B et al. 2017. Surface guided radiation therapy (SGRT): The sensitivity of the Region of Interest (ROI) selection on the translational and rotational accuracy for whole breast irradiation. Int. J. Radiat. Oncol. Biol. Phys. 99(2): E666-E667.

Heinzerling JH et al. 2020. Use of surface-guided radiation therapy in combination with IGRT for setup and intrafraction motion monitoring during stereotactic body radiation therapy treatments of the lung and abdomen. J. Appl. Clin. Med. Phys. 1-4. https:// doi.org/10.1002/acm2.12772.

Herron E, Murray M, Hilton L, Goldstein T, Ogunleye TB, Bailey D. 2018. Surface guided radiation therapy as a replacement for patient marks in treatment of breast cancer. Int. J. Radiat. Oncol. Biol. Phys. 102(3): e492-e493.

Kost S, Guo B, Xia P, Shah S. 2019. Assessment of setup accuracy using anatomical landmarks for breast and chest wall irradiation with surface guided radiation therapy. Pract. Radiat. Oncol. 9(4): P239-247.

Kügele M et al. 2019. Surface guided radiotherapy (SGRT) improves breast cancer patient setup accuracy. J. Appl. Clin. Med. Phys. 20 (9): 61-68.

Li G et al. 2011. Motion monitoring for cranial frameless stereotactic radiosurgery using video-based three-dimensional optical surface imaging. Med. Phys. 38(7): 3981-94.

Li G, Lovelock DM, Mechalakos J, Rao S, Della-Biancia C, Amols H, Lee N. 2013. Migration from full-head mask to "open-face" mask for immobilization of patients with head and neck cancer. J. Appl. Clin. Med. Phys. 14(5): 243-254.

Moser T, Creed M, Walker R, Meier G. 2020. Radiotherapy tattoos: Women's skin as a carrier of personal memory - What do we cause by tattooing our patients? Breast J. 26: 316-318.

Pham N-L et al. 2014. Frameless, real-time, surface imaging-guided radiosurgery: Update on clinical outcomes for brain metastases. Transl. Cancer Res. 3(4): 351-357.

Zagar TM et al. 2017. Utility of deep inspiration breath hold for leftsided breast radiation therapy in preventing early cardiac perfusion defects: A prospective study. Int. J. Radiat. Oncol. Biol. Phys. 97 (5): 903-909.

Cite this article as: Nguyen D, Farah J, Josserand-Pietri F, Barbet N, Khodri M. 2021. Benefits and challenges of standard ceiling-mounted surface guided radiotherapy systems for breast treatments on Halcyon ${ }^{\mathrm{TM}}$. Radioprotection 56(4): 295-301 